\title{
Les Eclogues sacrées prises du Cantique des Cantiques de Salomon et la poésie biblique de Remy Belleau
}

\author{
JANE COUCHMAN
}

Les Eclogues sacrées prises du Cantique des Cantiques de Salomon de Remy Belleau font partie de sa dernière oeuvre publiée. Elles figurent après Les Amours et nouveaux Eschanges des Pierres précieuses: vertus \& proprietez d'icelles et le Discours de la Vanité pris de l'Ecclésiaste de Salomon dans un volume publié en 1576, à peine six mois avant la mort du poète. ${ }^{1}$ Jean Dorat souligne dans le Tombeau de Belleau qu'il s'agit ici d'un chant du cygne digne du poète:

Dein \& Idumaei resonans pia cantica cycni Atque dei atque animae basia sancta pia. ${ }^{2}$

Voici le début du Cantique des Cantiques tel qu'il apparaît dans la Vulgate:

Osculetur me osculo oris sui

Quia meliora sunt ubera tua vino,

Fragrantia unguentis optimis.

Et voici la version de Belleau:

\section{L'Espovse}

Doncques mon cher Espous, mon mignon, ma chere ame En fin est de retour: que sa bouche de basme Me donne promptement pour ma flamme appaiser, Le nectar ensucré d'un amoureux baiser: Ton amour est plus doux, \& plus douce ta grace, Que le vin muscatel, encores qu'il surpasse Les plus souefues odeurs, \& les baisers mignars Animez de soupirs, qu'en baisant tu depars, Mieux fleurans que le thym, que la rose espanie, Et tout l'air emmusqué des parfums d'Arabie. 
On comprend peut-être pourquoi Henri Weber et tant d'autres critiques ont trouvé les Eclogues sacrées "déconcertantes". ${ }^{3}$ En les écrivant, Belleau aborde un problème qui date de l'établissement des canons bibliques hébreu et chrétien aux premiers siècles de notre ère: comment lire ce magnifique poème, comment le "traduire" pour ses contemporains, et comment traiter les tensions réelles ou apparentes entre l'amour érotique et l'amour spirituel dans l'oeuvre? Les solutions qu'apporte Belleau à ce problème sont intéressantes en ce qu'elles sont entièrement conventionnelles, qu'elles respectent et révèlent les goùts et le sentiment religieux de son époque et de son milieu. C'est ce qui rend les Eclogues sacrées "déconcertantes". Au lecteur moderne, que ses habitudes critiques soient formées chez Henri Weber et Henri Chamard ou même chez Frances Yates, Michel Jeanneret, et Terence Cave, ${ }^{4}$ la traduction peut paraître infidèle, les sentiments peu sincères, le langage trop précieux, et le tout mêlé d'étrange érudition et de naïveté, de sacré et de profane. Nous voulons suggérer ici que pour Belleau les Eclogues sacrées représentent une tentative d'intégration d'éléments dispersés jusqu'à ce point dans son œuvre, un chant du cygne digne non seulement de son projet poétique mais aussi du projet qu'il partage avec de nombreux autres poètes de marier le beau langage élevé de la nouvelle poésie aux sujets les plus sacrés.

Les Eclogues sacrées sont dédiées à la Reine Louise de Vaudemont, femme d'Henri III, très renommée pour sa dévotion à une cour où la dévotion est particulièrement admirée. ${ }^{5}$ Dans sa dédicace, Belleau soulève le problème de la tension entre langage érotique et amour spirituel:

Mais parce qu'en icelles ne se chante que d'amoureuses passions, \& que par aduenture quelques uns les pourroyent interpreter à leur aduantage, \& selon leur affection particuliere, à fin de ne tomber en ceste erreur, i'ay bien voulu les aduertir, que c'est vn amour tout diuin \& tout spirituel, par lequel on peut iuger l'heur, la felicité, et le souuerain bien, qui prouient d'estre estroittement vni par viue \& ardente amour auec l'Eglise \& IESVSCHRIST, figuré sous le nom de l'Espous, et l'Eglise sous le nom de l'Espouse: discourant ensemble humainement de la douceur de leurs baisers, de leurs chastes $\&$ parfaittes amours, embrassemens, graces, \& de leurs rares \& immortelles beautez, comme vous pourrez voir plus aisément, par les petits arguments que i'ay mis sur chacune Eclogue, où n'y a rien qui ne soit saint \& diuin, \& digne des chastes oreilles d'une grande Royne, telle que vous estes.

$$
\text { II, 297) }
$$

Voilà deux exemples de ces "arguments":

\section{ECLOGVE II.}

L'Eglise se vante estre belle comme la fleur, fraische comme la rose, tendre comme les lis qui croissent au fond des vallees, desire ardemment prendre 
son repos sous l'ombre des ailes de IESUS-CHRIST son espous.

(II, 303)

\section{ECLOGVE III.}

L'Eglise sous la figure de l'ame pecheresse, estant pressee du sommeil d'ignorance, \& sommeillant és tenebres de peché, cherche IESUSCHRIST au hasard \& danger de sa vie.

(II, 306)

Comme on le sait, l'exégèse du Cantique des Cantiques présente un intérêt tout particulier, étant donné que les commentateurs passent directement aux niveaux allégorique, tropologique et anagogique, refusant l'expression littérale de l'amour humain. ${ }^{6}$ Déjà avec Origène et encore au XII' ${ }^{\mathbf{e}}$ siècle avec Bernard de Clairvaux cette exégèse se situe dans le courant mystique et néo-platonicien du christianisme, courant renforcé chez Belleau et les autres poètes de la Pléiade par leur admiration pour l'Académie florentine. Au Moyen Âge et à la Renaissance, le langage du Cantique des Cantiques est interchangeable: les images sensuelles du Cantique sont transposées dans des poèmes profanes, mais en même temps le langage de la contemplation extatique se nourrit de la poésie érotique. Ceci pose un problème à la fois pour la critique laïque qui y voit un manque de sincérité et pour la critique chrétienne qui y voit un danger. ${ }^{7}$ Mais pour Belleau et ses contemporains le problème se définit autrement. Pour eux, la définition la plus claire, comme Belleau l'indique déjà dans son Commentaire au deuxième livre des Amours de Ronsard, se trouve chez Marsile Ficin dans son Commentaire au Banquet de Platon. ${ }^{8}$ Nous y lisons une description philosophique, théologique, scientifique, entièrement compatible avec la vision médiévale. Seul à éviter (nous en reparlerons) est l'amour vulgaire par lequel "l'homme se trouve rabaissé au rang de la bête". L'homme doit chercher inlassablement l'amour divin, car, "par l'amour divin l'homme s'élève au-dessus de sa propre nature et monte en Dieu".9

Quand, dans sa dédicace à la Reine, Belleau souligne le caractère sacré de ces poèmes d'amour, ce n'est ni par "prudence", ni par "naïveté" 10 mais pour se placer dans cette tradition et pour rappeler à sa lectrice, et aux lecteurs en général, un lieu commun de l'herméneutique qu'ils connaissent tous pour l'avoir entendu en maint sermon, mainte méditation, maint poème religieux, tant protestant que catholique, soit dit en passant. ${ }^{11}$ Docte parmi les doctes, Belleau n'est pas moins sensible aux traditions et aux sources en matière de poésie religieuse qu'il ne l'est dans les domaines où nous le connaissons mieux, la pastorale de sa Bergerie et la poésie scientifique de ses Pierres 


\section{2 / Renaissance and Reformation}

précieuses. Nous pouvons prendre tout à fait au sérieux le projet du poète de présenter, dans un style apte à être reçu par son public particulier (la famille royale, la famille des Guises) comme par le public en général, un poème pieux.

En "traduisant" le poème pour son public, Belleau reste fidèle à beaucoup d'éléments essentiels de l'original. Il suit exactement l'ordre du texte biblique. Les huit "éclogues" correspondent aux huit chapitres bibliques. Le poète maintient la forme d'un dialogue dramatique entre l'Époux et l'Épouse. Il garde aussi les images pastorales de l'original. L'amant est un pasteur, l'amante une vigneronne, mais déjà en hébreu la pastourelle est aristocratique, car le pasteur est le roi Salomon et la vigneronne la reine de Saba. Lelieu était bucolique dans l'original, et partage ici comme dans mainte autre version les qualités du locus amoenus et de l'Arcadie classiques, et celles du paradis terrestre biblique et du jardin clos médiéval. Belleau retient aussi les images de flore et de faune qu'emploient les amants pour se décrire. Et il rehausse la présentation d'un amour qui mène à une consommation extatique. Toutes les ressources du traducteur d'Anacréon et de l'auteur de la Bergerie sont mises au service de la paraphrase biblique.

Les apports spécifiques et nouveaux de Belleau n'en sont pas moins frappants, surtout en ce qui concerne l'amplification et l'introduction dans le poème de topoï et de genres conventionnels de l'époque. La version des premiers vers du Cantique citée plus haut en présente un excellent exemple. Les quatorze mots de la Vulgate sont transformés en dix vers, la syntaxe simple et directe de l'original devient complexe, recherchée. "Qu'il m'embrasse" devient "que sa bouche ... me donne ... le nectar ... d'un baiser," ce qui laisse toute la place aux modificatifs ("de basme", "promptement", "pour ma flamme apaiser", "ensucré", "amoureux"). On peut comparer ce vers à la traduction du même passage dans les "Amours de Salomon et de la Sunamite" du poète protestant Jean-Baptiste Chassignet, texte tout aussi lié au goût de l'époque d'ailleurs.

Que de mille baisers de sa bouche embasmée

Il restaure mon coeur Celui que j'aime tant.

O Roi de mes désirs il n'est vin qui autant

Que tes sucrez amours plaise à ta bien-aimée. ${ }^{12}$

Chez Belleau, lisons un autre passage célèbre en latin: "Nigra sum, sed formosa, filiae Jerusalem" (I:4)

Ie suis noire vrayment, vous le voyez, Fillettes, De la sainte Cité citoyennes Nymphettes,

Mais ce teint brun pourtant n'efface la beauté

Qui reluist sur ma face en grave majesté.

(II. 300-301) 
Il est déjà évident que les amplifications par Belleau du Cantique des Cantiques sont riches des conventions et des ornements de son époque. C'est un exemple du style "doux-coulant" de La Pléiade, et du style "mignard" de Belleau tout particulièrement. ${ }^{13} \mathrm{Ce}$ style est caractérisé par l'emploi des diminutifs, souvent à la rime (Fillettes / Nymphettes; mollette / tendrelette). On y trouve beaucoup de descriptions "précieuses" avant la lettre, comme dans l'exemple suivant:

Capilli tui sicut greges caprarum

Quae ascenderunt de monte Galaad

(III:i)

Et paroissent ainsi les tresses vagabondes

De tes cheveux retors \& repliez en ondes,

Que la molle toison de ce ieune troupeau,

De ce troupeau barbu qui nourrist sur sa peau

Le poil blanc \& frisé d'ondoyantes crespines

Sur les tapis herbus des croupes Galadines.

(II. 308)

Aux traditions amoureuses d'Ovide, de Ficin et de Pétrarque la version de Belleau doit plusieurs conventions, en particulier l'image des yeux qui transmettent les flammes d'amour. Les amants bibliques ne brûlent pas, mais des flammes apparaissent chez Belleau dès le troisième vers; la traduction des vers suivants est frappante:

Averte oculos tuos a me, quia ipsi me avolare fecerunt

(VI:5)

Hà, ie brusle d'Amour! Hà, ie brusle, ma Belle,

Détourne tes beaux yeux, qui font que je chancelle

Esblouy de leur grace $\&$ de leur vive ardeur

Tant me rendent honteux et m'abaissent le cueur.

(II. 316)

En analysant le Cantique des Cantiques en hébreu on a pu y trouver une série de poèmes de différents genres, ce que l'on trouve aussi chez Belleau. Comme tant d'autres poètes avant lui, Belleau fait sur le passage "Surge, propera, amica mea, columba mea, formosa mea et veni ..." (II:10) une belle chanson du lever du jour:

\section{L'Espovs}

Maistresse levez-vous, sus donc hastez le pas,

Ma Colombe, mon coeur, mon miel, mon doux appas,

Venez avecques moy, suyuez moy à la trace.

L'Hyuer morne de froid, blanc de nege \& de glace 


\section{4 / Renaissance and Reformation}

S'est derobé de nous, \& l'Astre pluuieux

Se plongeant a faict place au printemps gracieux:

La Terre de couleurs \& de fleurs bigarree

Descouure son beau sein, \& sa robe pourpree,

Espandant ses thresors: c'est la belle saison

Qu'il faut tailler la vigne, et laisser la maison

Pour habiter les champs: desia la Tourterelle

Dessus cest arbre sec redouble sa querelle...

(II. 304)

Le goût de Belleau pour la description des bijoux paraît dans le poème suivant, basé sur le latin:

Pulchra sunt genae tuae sicut turtures

Colum tuum sicut monilia

Murenulas aureas faciemus tibi

Vermiculatas argento

(I:9 \& 10)

\section{Et Belleau:}

Le teint frais et douillet de ta face vermeille

Rougist estincelant sous deux pendans d'oreille,

Tout ainsi que l'Aurore: \& l'yuoire poli

De ton col blanchissant se presente anobli

De perles, de rubis, \& de pierres exquises

Dans le fond d'un carquan naifuement assises.

Ie te doray encor un autre riche attour,

Qui fera pour jamais tesmoing de nostre amour:

Deux bracelets d'or fin taillez en Damasquine,

Une chaisne, un carquan, et de soye plus fine

Un tissu marqueté de beaux gros boutons dor

Mis en oeuvre d'espargne, $\&$ des bagues encor.

(II, 302)

Ne se limitant pas aux bijoux, Belleau compose aussi le "blason" du corps de l'amante,

De ton sein relevé l'enfleure aboutissante

D'une framboise tendre, à demi rougissante.

(II. 309)

Il n'y manque pas le "baiser" de la première strophe, dont la parenté avec ceux de la Bergerie est évidente: en écrivant "Mouches qui massonnez les voûtes encirées ..." Belleau associe également le baiser aux épices, aux fleurs parfumées, aux flammes. (II, 86)

Quand, pour décrire les amours divines de Jésus-Christ et de son Église, 
Belleau emploie des formes, des images, un langage qui paraissent à la critique plus propres à la poésie profane, il reste fidèle à son modèle biblique, aussi bien qu'à son propre projet poétique et au projet plus vaste de réconciliation entre la poésie humaniste et la poésie chrétienne, afin de doter cette dernière de tous les attraits du premier. Parmi de nombreux contemporains de Belleau mentionnés en particulier par Terence Cave, citons-en un, Belleforest, qui défend ainsi le projet: "Ce ne sont des délices et folles mignardises que nous avons traitées jadis en nos livres n'y des sujets d'une vaine éloquence, ains y est le style doux, coulant et plein de sainte affection, ravissant les âmes à la contemplation de Dieu."14

L'engagement biblique de Belleau date du début de sa carrière, de son premier poème publié, qui accompagne les Cantiques du premier advenement de Jésus-Christ de son ami le poète et peintre Nicolas Denisot (1553). ${ }^{15}$ Ici Belleau établit déjà le contraste entre la poésie d'amour profane ("variable", "fabuleux") et la poésie d'amour chrétien ("éternel et durable").
Le sujet n'est point d'Amours
Le trait n'est point variable
Ny fabuleux le discours
Mais éternel et durable.
...
Icy l'on voit seulement
Descouvertes les merveilles
Du sacré Advenement
Digne des Sainctes oreilles...

(II, 454)

Voilà déjà soulevée la question de la destination des Eclogues sacrées, question que Belleau pose à plusieurs reprises et sous plusieurs formes, comme dans un curieux sonnet "D'un bouquet envoyé le mercredi des cendres" et dans un poème "Contre l'amour", où il regrette d'avoir chanté l'amour-passion,

... car ce n'est qu'une erreur

Qui glisse en nous, \& comme par le songe

Naist un plaisir qui s'escoule en mensonge.

$(I, 171)$

"Tu n'es point Dieu", dit le poète à Cupidon; c'est de nous-mêmes que vient le mal que nous essayons de glorifier en l'attribuant à un dieu. Belleau prie plutôt le "ciel" et les "saintes divinités" de le protéger de ce "cruel orage". Expressions conventionnelles, dira-t-on avec raison, mais que Belleau emploie toujours avec sérieux, faisant suivre ce poème d'une "Prière à Dieu" débutant par l'image maritime sur laquelle termine le poème précédent: 


\section{6 / Renaissance and Reformation}

Sus sus mon âme, avant gaignons le port

Nous sommes forts car Dieu est notre fort...

$(\mathrm{I}, 172)$

L'inspiration biblique et chrétienne n'a donc pas manqué au poète, mais c'est à partir de la deuxième édition, et de la Seconde Journée de la Bergerie en 1572 que paraissent les grandes versions des textes bibliques. La Seconde Journée de la Bergerie s'ouvre sur une série de très belles prières tirées du livre de Job et rendues dans un style admirablement sobre et simple.

L'homme nay de la femme, en viuant peu de temps

Est plein de mille maux \& de mille tourmens

Il est comme la fleur qui naissant est coupée

Et fuit ainsi que l'ombre, \& n'a point de durée.

(II, 8)

Belleau dispose donc d'une variété de registres poétiques, et s'il choisit le style "mignard" pour ses Eclogues sacrées c'est parce que ce style s'accorde pour lui et pour son public avec la matière du Cantique.

Il n'est pas étonnant que le poète choisisse, pour commencer sa Journée, d'adresser ses "humbles prières à ce grand Dieu, autheur de tout bien, plein de vérité, de justice $\&$ de miséricorde, suyvant d'heureuse mémoire des complaintes et doléances de ce bon Job," (II, 5) car ces mêmes prières pénitentielles figurent à l'Office des morts et s'emploient de façon régulière dans la prière personnelle pour rappeler au chrétien son état mortel et misérable devant Dieu. Seule variante, dans l'ordre de la contemplation formelle: ses prières se placent dans la méditation du soir, tandis que la prière du matin célèbre normalement la vie et le sacrifice du Christ. ${ }^{16}$ Dans la poésie biblique de Belleau il n'y a aucune référence directe au Christ ni à sa vie. Les références figurales (à Job, à David, à l'Époux) sont fréquentes.

La Seconde journée de la Bergerie se termine également sur une paraphrase biblique, le récit des "Amours de David et de Bersabée", poème qui, comme les Eclogues sacrées, embarrasse quelque peu la critique. ${ }^{17}$ Le poète reprend dans une version assez directe le passage du livre de Samuel (II, 11 et 12) décrivant leurs amours, la colère de Dieu, et le repentir de David sous l'influence du prophète Nathan. Deux changements qu'apporte Belleau à la version biblique sont révélateurs: l'intervention du petit dieu d'amour, et un dialogue allégorique entre la Justice et la Clémence de Dieu afin de décider du sort du roi égaré. Ces deux éléments signalent à mon avis une vraie lutte entre l'amour-passion et l'amour chrétien. Le petit dieu d'amour païen, un peu comme Satan dans l'histoire de Job, "veut tenter sa force" et réussit à vaincre le grand roi David, l'élu de Dieu, homme par ailleurs sage et puissant. La façon dont David est atteint de cet amour aussi bien que les 
symptômes dont il souffre viennent directement de la description de l'amour vulgaire ou bestial chez Ficin. ${ }^{18}$

Des yeux, ce trait doré entra iusqu'au dedans

Du foye $\&$ du poulmon, \& de mouchons ardans

Luy rechauffe le sang, \& de nouvelles peines

Luy trouble le cerueau, receut dans ses veines

Vn ulcere, vn venin, vn feu qui va bruslant...

(II, 142)

Le comportement de David sous l'influence de Cupidon est nettement criminel. La juste colère de Dieu le mènerait à se venger de l'amour païen en punissant de mort sa victime. C'est la Clémence de Dieu qui trouve moyen de transcender le faux amour en accordant à David la possibilité du repentir. ${ }^{19}$ Et c'est sur les psaumes pénitentiels de David que se termine la Bergerie:

Ayant fait ces regrets, prend sa lyre d'yvoire Bagne ses yeux de pleurs, sacrant à la mémoire De son pêché commis, les larmes \& les sons Et les vers animez de ses tristes chansons. (II, 151)

Belleau fait donc sortir de la victoire sur l'amour païen les psaumes pénitentiels de David / Orphée, vraie poésie d'amour. Tout se rejoint en un sens, car, quoique Belleau n'y fasse pas allusion, de l'union de David et de Bethsabée est né un fils, Salomon, porte-parole de Dieu dans l'Ecclésiaste et dans le Cantique des Cantiques. Et dans ce sens, la Seconde Journée de la Bergerie est circulaire, s'ouvrant et se fermant sur des prières pénitentielles bien connues de la tradition chrétienne. C'est un cercle qui se ferme pour se rouvrir sur l'avenir, car le pêcheur qui fournit le poème de "David et de Bersabée" le destine, avec la "Complainte ... sur la mort de Joachim du Bellay" qui le précède, à une lecture matinale "le lendemain" et c'est par impatience seulement que les figurants de la Bergerie les lisent la nuit même. (II, 133)

Dans sa poésie biblique, Belleau passe de la pénitence de Job et de David à la contemplation de la vanité de toute chose avec l'Ecclésiaste. ${ }^{20}$ Encore une fois, Belleau suit de près le texte biblique, en y ajoutant toutefois deux passages d'intérêt personnel, dont l'un porte sur la vanité des études scientifiques: "J'ai discouru, savant, les Astres radieux ... "(II, 265) et l'autre sur la vanité des palais et des jardins: "J’ai planté, j’ai semé, j’ai fait le jardinage ..." (II, 267). La lecture de l'Ecclésiaste se termine sur une invitation à contempler 1 'amour divin: 
Doncques souvienne toy des graces du Seigneur, Pendant que ta iouuence est en sa prime fleur...

(II, 292)

Les Eclogues sacrées se trouvent bien à leur place au sommet d'un développement spirituel tout à fait rigoureux selon les termes de la spiritualité de la Contre-Réforme et d'un projet esthétique, partagé avec Denisot, Belleforest et bien d'autres, et mettant le plus beau langage au service du Dieu d'amour chrétien par opposition au dieu d'amour païen.

À côté du traducteur humaniste, du peintre de la nature, et du poète scientifique, il faut admettre l'existence légitime d'un Remy Belleau poète biblique, caractérisé ici à grands traits. Si les Eclogues sacrées nous paraissent toujours un peu déconcertantes, on peut y reconnaître la tension qui existe chez Belleau entre les différentes expressions de l'amour au seizième siècle. Mais Belleau chante trop bien les préoccupations parfois contradictoires de ses contemporains pour que l'on s'attende à une synthèse entièrement cohérente de ses diverses voix.

\section{York University}

Notes

1 Nous avons utilisé les Euvres poétiques de Remy Belleau, éd. Ch. Marty-Laveaux, 2 t. (Genève: Slatkine, 1965). (Réimpression de l'édition de 1877-78.) La formule (II, 297) qui suit une citation de l'œuvre de Belleau en indique le tome et la page. Voir aussi les CEuvres complètes de Remy Belleau, éd. A. Gouverneur (Paris: A. Franck, 1867). Édition princeps des Amours et nouveaux Eschanges des Pierres précieuses: vertus proprietez d'icelles, du Discours de la Vanité pris de l'Ecclésiaste de Salomon et des Eclogues sacrées prises du Cantique des Cantiques de Salomon, (Paris: Mamert Patisson au logis de Robert Estienne, 1576).

2 Belleau, CEuvres, éd. Gouverneur, t. III. p. 366. Luigi Monga, dans son Genre Pastoral au XVTe siècle (Paris: Éditions Universitaires, 1947), p. 117, constate que ces vers s'appliquent aux "baisers" de la Seconde Journée de la Bergerie, ce qui aurait provoqué une relecture exégétique de toute l'œuvre de Belleau. Mais étant donné la référence au chant du cygne et à la lecture allégorique conventionnelle du Cantique des Cantiques, les vers ne peuvent se rattacher qu'aux Eclogues sacrées.

3 Henri Weber, La Création poétique au XVI' siècle en France (Paris: Nizet, 1955) t. I, p. 284; voir aussi Henri Chamard, Histoire de la Pléiade (1939; Paris: Didier, 1961), t. III, p. 268; et Luigi Monga, op. cit., pp. 74ff, p. 102.

4 Frances A. Yates, The French Academies of the Sixteenth Century (London: The Warburg Institute, 1947). Michel Jeanneret, Poésie et tradition biblique au XVI siècle (Paris: Corti, 1970). Terence C. Cave, Devotional Poetry in France (Cambridge: The University Press, 1969).

5 Cf. F. Yates, op. cit., p. 157. et T.C. Cave, op.cit., p. 11.

6 Voir Henri de Lubac, Exégèse médiévale (Paris: Aubier, 1962), surtout la Première partie, pp. 586ff.

7 J. Bruckmann et J. Couchman, “Du 'Cantique des Cantiques' aux 'Carmina Burana': amour sacré et amour érotique”, dans L'Érotisme au moyen-âge (Montréal: Édition de l'Aurore, 1977). Cf. Anders Nygren, Eros und Agapé: Gestaltwandlung der Christliche Liebe (Gutersloh: C. Bertelsmann, 1930-37), vol. II, p. 71, et E. Gilson, La théologie mystique de St. Bernard (Paris: Vrin, 1947), Appendice IV: "St. Bernard et l'amour courtois", p. 215. 
8 Pierre de Ronsard, Les CEuvres (Paris: Nicolas Buon, 1609). Première édition avec le commentaire de Belleau, 1560. Plusieurs références, à l'amour "d.-inement interprété" (p. 141) et à l'amour-maladie (p. 169) entre autres.

9 Marsile Ficin, Commentaire sur le Banquet de Platon, traduit par Raymond Marcel (Paris: Les Belles Lettres, 1956), pp. 256-257.

10 H. Weber, op. cit., p. 284, note 1.

11 T.C. Cave, op. cit.,p. 2,p. 263; cf. Lily B. Campbell, Divine Poetry and Drama in Sixteenth-Century England (Cambridge: The University Press, 1955), pp. 55ff.

12 Cité par Albert-Marie Schmidt, Études sur le XVI siècle (Paris: Albin Michel, 1967), p. 213. Le poème date de 1592 mais n'a pas été publié.

13 A. Eckardt, op. cit., pp. 154-159. H. Chamard, op. cit., p. 268, "La note amoureuse et même sensuelle de l'œuvre hébraïque a conduit trop souvent le nouveau traducteur à verser outre mesure dans ce qui fut sont défaut habituel, la mignardise." H. Weber,op. cit., p. 275. Belleau luimême emploie souvent le mot "mignardise" pour montrer son admiration du style de Ronsard.

14 Cité par T.C. Cave, op. cit., p. 63.

15 Le Conte d'Alsenois, Cantiques du premier advenement de Jésus-Christ (Paris: chez la Veuve Maurice de la Porte, 1553).

16 T.C. Cave, op. cit., p. 25 , p. 48.

17 Euvres éd. Marty-Laveaux, t.II, pp. 138ff. Dans La Bibliothèque française (1948) t. xii, pp. 291301 ; l'abbé Goujet le traite de "pièce impertinente". Cité avec approbation par Alexandre Eckhardt, Rémy Belleau, sa vie, sa "Bergerie"(Budapest: Nâmeth, 1917), p. 94. R.A. Sayce, The French Biblical Epic in the Seventeenth Century (Oxford: Clarendon Press, 1955), p. 36, trouve qu'il y a "a serious ambiguity" dans cette juxtaposition de Cupidon avec le Dieu des chrétiens, ce qu'il décrit comme "characteristic of the Pléiade's attitude to pagan and Christian merveilleux."

18 Marsile Ficin,op. cit.,pp. 246-247, l'amour vulgaire entre par les "fenêtres vitrées" des yeux et la contagion devient "la peste la plus grave de toutes", en "agitant le sang".

19 R.A. Sayce, op. cit., pp. 36-37. Pour Sayce, cette allégorie "was no doubt intended as a substitute for the council of the gods in classical epic."

20 T.C. Cave, op. cit., p. 44, pp. 96-97. 